\title{
Ascertaining severe perineal trauma and associated risk factors by comparing birth data with multiple sources
}

\author{
Amanda J Ampta,b and Jane B Ford ${ }^{a}$ \\ ${ }^{a}$ Clinical and Population Perinatal Health Research, Kolling Institute, University of Sydney, NSW, Australia \\ b Corresponding author: amanda.ampt@sydney.edu.au
}

\section{Article history}

Publication date: September 2015

Citation: Ampt AJ, Ford JB. Ascertaining severe perineal trauma and associated risk factors by comparing birth data with multiple sources. Public Health Res Pract. 2015;25(4):e2541544. doi: http://dx.doi. org/10.17061/phrp2541544

\section{Key points}

\section{- Severe perineal trauma can be} ascertained by birth or by linked data, but ascertainment increases when a linked dataset is used compared with birth data alone

- Agreement between the datasets is higher for data collected since 2006; however, possible underreporting by birth data remains a concern

- Differences between linked data and birth data translate into different risk estimates of severe perineal trauma for episiotomy

- Investigation should be ongoing, with further studies exploring data quality as new data become available

\section{Abstract}

Objectives: Population data are often used to monitor severe perineal trauma trends and investigate risk factors. Within New South Wales (NSW), two different datasets can be used, the Perinatal Data Collection ('birth' data) or a linked dataset combining birth data with the Admitted Patient Data Collection ('hospital' data). Severe perineal trauma can be ascertained by birth data alone, or by hospital International Classification of Diseases Australian Modification (ICD-10-AM) diagnosis and procedure coding in the linked dataset. The aim of this study was to compare rates and risk factors for severe perineal trauma using birth data alone versus using linked data.

Methods: The study population consisted of all vaginal births in NSW between 2001 and 2011. Perineal injury coding in birth data was revised in 2006, so data were analysed separately for 2001-06 and 2006-11. Rates of severe perineal injury over time were compared in birth data alone versus linked data. Kappa and agreement statistics were calculated. Risk factor distributions (maternal age, primiparity, instrumental birth, birthweight $\geq 4 \mathrm{~kg}$, Asian country of birth and episiotomy) were compared between women with severe perineal trauma identified by birth data alone, and those identified by linked data. Multivariable logistic regression was used to calculate the adjusted odds ratios (aORs) of severe perineal trauma.

Results: Among 697202 women with vaginal births, 2.1\% were identified with severe perineal trauma by birth data alone, and $2.6 \%$ by linked data. The rate discrepancy was higher among earlier data (1.7\% for birth data, $2.4 \%$ for linked data). Kappa for earlier data was 0.78 (95\% Cl 0.78, 0.79), and 0.89 $(95 \% \mathrm{Cl} 0.89,0.89)$ for more recent data. With the exception of episiotomy, differences in risk factor distributions were small, with similar aORs. The aOR of severe perineal trauma for episiotomy was higher using linked data (1.33, $95 \% \mathrm{Cl} 1.27,1.40)$ compared with birth data $(1.02,95 \% \mathrm{Cl} 0.97,1.08)$.

Conclusions: Although discrepancies in ascertainment of severe perineal trauma improved after revision of birth data coding in 2006, higher ascertainment by linked data was still evident for recent data. There were also higher risk estimates of severe perineal trauma with episiotomy by linked data than by birth data. 


\section{Introduction}

Major perineal tearing can occur during childbirth when a spontaneous tear or an episiotomy extends to include the anal sphincter musculature (third-degree tear), or also the anal mucosa (fourth-degree tear). ${ }^{1}$ These tears are collectively known as severe perineal trauma and are distressing adverse outcomes of some vaginal births, with short-term and potential long-term consequences. ${ }^{1-3}$ Rates of severe perineal trauma are increasing in New South Wales (NSW) ${ }^{4}$ and internationally. ${ }^{5-8}$ Identifying and monitoring severe perineal trauma is a gauge of safe and appropriate care, and is included as one of 55 quality indicators by the Australian Institute of Health and Welfare. ${ }^{9}$ Population health data, with their advantage of no sampling bias, are often used to monitor trends of severe perineal trauma and assess their association with risk factors. 4,5,7,10 The accuracy and reliability of population health data are typically reported by validation studies to assess data quality. ${ }^{11-15}$

Severe perineal trauma can be ascertained from two different population datasets in NSW: the NSW Perinatal Data Collection (PDC, or 'birth' data), and the Admitted Patient Data Collection (APDC, or 'hospital' data). Both datasets have information relating to the same deliveries, and a researcher has the option of using either dataset or a combination of both. Because the hospital data contain information regarding all hospitalisations, both maternity and nonmaternity related, the hospitalisations relating to the births need to be identified. If a researcher uses hospital records alone, some of the maternity hospitalisation records may be missed. ${ }^{16}$ Linkage between the hospital and birth datasets can accurately identify births and associated complications. ${ }^{17} \mathrm{De}-$ identified information from both datasets can be combined into a single record for each birth. Although birth data alone can be used for research, combining it with hospital data provides more detailed information regarding diagnoses and procedures, and can increase the reliability and accuracy of reporting around labour and delivery. ${ }^{13}$ However, linked data are not typically available until 12-18 months after the birth data, so there is a slight trade-off between timeliness and reliability or accuracy.

By its nature, a linked dataset is more complex because some clinical information will be available from multiple sources and in different formats. Once the data sources have been linked, severe perineal trauma can be identified from the birth data or hospital data, or a combination of both. A previous validation study has shown that identification from hospital data alone, with reporting by either the Australian Classification of Health Interventions $(\mathrm{ACHI})$ procedure code (16573-00, suture of third or fourth tear of the perineum) or International Classification of Diseases Australian Modification (ICD10-AM) diagnosis codes (O70.2, third-degree perineal laceration; or O70.3, fourth-degree perineal laceration) is the most reliable and accurate method of ascertaining the incidence in NSW. ${ }^{13}$ Some Australian studies have ascertained severe perineal trauma by this method ${ }^{4,18,19}$ and others have used birth data. ${ }^{20,21}$

Some risk factor variables will be in both data collections - for example, episiotomy and instrumental birth. A linked dataset gives researchers the opportunity to use those variables from the most reliable and accurate source, as evidenced by previous validation studies. The aim of this study is to compare the rates and risk factors for severe perineal trauma, using both birth data alone and linked data.

\section{Methods}

\section{Data sources}

The study population consisted of all vaginal births in NSW from 2001 to 2011, with de-identified information provided in a linked dataset containing data from the NSW PDC (birth data) and the APDC (hospital data). The PDC is a statutory collection of all NSW hospital and home births occuring at $\geq 20$ weeks gestation or $\geq 400 \mathrm{~g}$ birthweight, and includes information on maternal characteristics; medical and obstetric information; and labour, birth and infant condition. ${ }^{22}$ Information is recorded by the attending midwife or doctor. The APDC is a census of all hospital admissions and is collected from the hospital medical record after a patient has been discharged. Each record relates to a single hospital admission. In addition to administrative and demographic data, the hospital data contain clinical diagnosis and procedure information, which have been coded according to the ICD-10-AM ${ }^{23}$ and the ACHI. ${ }^{24}$ In this study, each linked record contained information from both data collections for each woman - birth data from the PDC and hospital data from the APDC. Linked data were from probabilistically linked birth and hospital data. Where a multiple pregnancy occurred, we included data pertaining to the firstborn.

The birth data collection form was revised in 2006, and recording of perineal status changed during the study period (Figure 1). Using the older version of the form, a woman with severe perineal trauma and an episiotomy would be coded as 'both tear and episiotomy', and so would a woman with a less severe tear and episiotomy. The newer form separated information regarding spontaneous tearing and episiotomy into two separate variables, distinguishing severe perineal trauma from other tears in the presence of an episiotomy. Both versions were in use during the 2006 calendar year because individual hospitals introduced the new form at different times. We have defined data collected using the older form as 'earlier data' (all pre-2006 data and data collected using the older form during 2006), and data collected on the newer form as 'more recent data' (data collected using the newer form during 2006 and post2006 data). In contrast to birth data, the diagnosis and 
Figure 1. Versions of the birth data collection form
(a) Older form
(b) Newer form
(pre-2006 revision)
(post-2006 revision)

\begin{tabular}{|c|c|}
\hline Perineal status & Perineal status \\
\hline Intact $\square$ & Intact $\square$ \\
\hline 1st deg. tear/graze $\square$ & 1st deg. tear/graze $\square$ \\
\hline 2nd deg. tear $\square$ & 2nd deg. tear $\square$ \\
\hline 3rd deg. tear $\square$ & 3rd deg. tear $\square$ \\
\hline 4th deg. tear $\square$ & 4th deg. tear $\square$ \\
\hline Episiotomy $\square$ & Other $\square$ \\
\hline Both tear and episiotomy $\square$ & Episiotomy \\
\hline Other $\square$ & Yes $\square$ \\
\hline & No $\square$ \\
\hline
\end{tabular}

procedure codes in the hospital data did not change and, in keeping with the results of a previous validation study ${ }^{13}$, we identified severe perineal trauma from the linked data by ICD-10-AM O70.2 or O70.3, or procedure coding 16573-00. Thus our comparison was between severe perineal trauma ascertained by hospital data alone within the linked data, and birth data alone.

\section{Analysis}

To compare the sources of severe perineal trauma identification, we calculated the rates over time for birth data alone, and for linked data. Kappa and agreement statistics were then used to compare severe perineal trauma identified from birth and from linked data, for both periods of data collection. Discordant cases were described. Because women with a diagnosis code for either a third-degree or a fourth-degree tear (severe perineal trauma) would require a suturing procedure, agreement would be expected between a diagnosis and a procedure code. We investigated if any discrepancy existed by calculating the agreement and Kappa statistics between these codes.

To ascertain the impact of data source on risk estimates, we compared the distributions of maternal age, primiparity, instrumental birth, infant birthweight $\geq 4 \mathrm{~kg}$, Asian maternal country of birth and episiotomy - all known to be associated with severe perineal trauma $a^{4,25}$ for two scenarios: (a) using birth data alone, and (b) using linked data. Information regarding parity, birthweight, instrumental birth and country of birth were identified from birth data, and episiotomy was identified if it was present in either birth or hospital data. ${ }^{13}$ Distributions of risk factors from the discordant records were also examined.

Two multivariable logistic regression models were then built, one for each scenario. The adjusted odds ratios (aORs) of severe perineal trauma for risk factors were calculated for each scenario and compared. We restricted analyses of risk factors to the more recent data, because the older collection form could not discriminate severe perineal trauma occurring with or without episiotomies. Because episiotomy has previously been reported as varying in association with severe perineal trauma according to type of delivery ${ }^{4,7}$, we also performed a sensitivity analysis comparing the aORs for women with episiotomies for delivery type (noninstrumental, forceps and vacuum)

SAS (version 9.3, SAS Institute, Cary, NC, USA) was used for all analyses. This study was approved by the NSW Population and Health Services Research Ethics Committee.

\section{Results}

Among women who had vaginal births between 2001 and $2011(N=697$ 202), rates of severe perineal trauma differed between birth data and linked data (Figure 2). Overall, the rate was $2.1 \%$ ascertained by birth data, and $2.6 \%$ by linked data. Differences in ascertainment are reflected in a relative increase in rates from 2001 to 2011 of $115.4 \%$ in birth data and $58.5 \%$ in linked data. The severe perineal trauma rate recorded by the earlier version of the birth data form was $1.7 \%$, compared with a rate of $2.4 \%$ recorded by linked data for the same women. This discrepancy decreased with the introduction of the newer form, with a birth data rate of $2.5 \%$ and a linked data rate of $2.9 \%$.

Figure 2. Severe perineal trauma rates among vaginal births from birth data and linked data

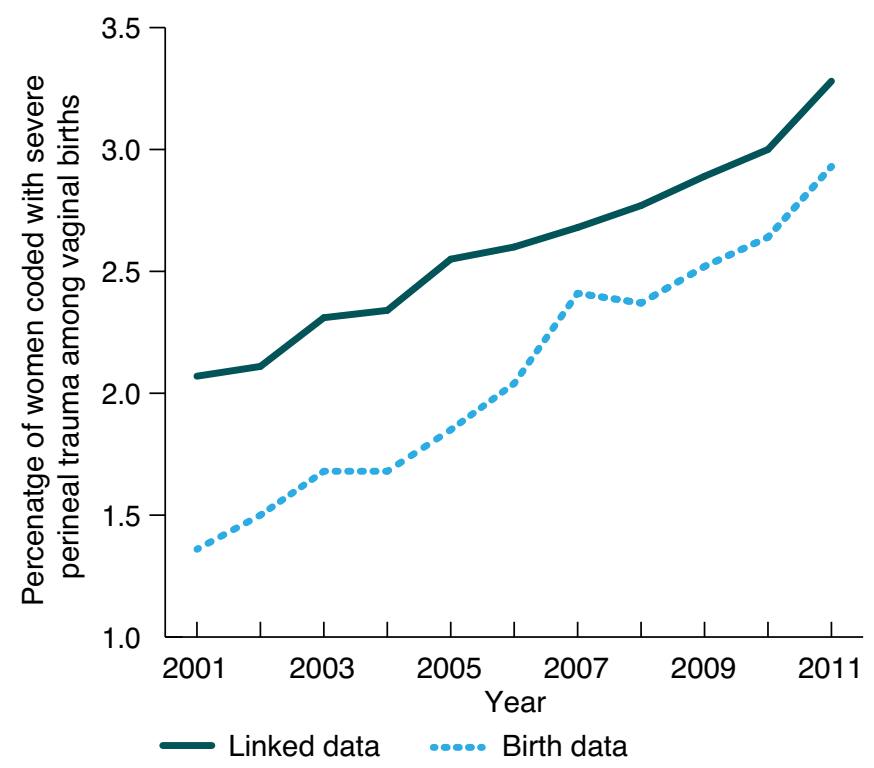

Within the linked data between 2001 and 2011, agreement was high between diagnosis and procedure coding (agreement 99.8\%; Kappa $=0.96,95 \% \mathrm{Cl} 0.95$, 0.96). There were 1474 discordant cases $(0.2 \%$ of total 
births). There were 1109 women coded with a third- or fourth-degree tear diagnosis but not with the associated suturing procedure (6.2\% of diagnosed tears), and 365 women had a suturing code but no diagnosis code (2.1\% of recorded suturing procedures).

As reflected in the rates, agreement between linked and birth data coding was lower among the earlier data (Kappa $=0.78,95 \% \mathrm{Cl} 0.78,0.79)$ than the more recent data (Kappa $=0.89,95 \% \mathrm{Cl} 0.89,0.89)$ (Table 1). Among the 8242 women with severe perineal trauma identified by linked data in the earlier data, 2679 (32.5\%) were discordant cases in which women were not coded as having severe perineal trauma by birth data. Among these, 1082 (40.4\%) had been coded as 'both tear and episiotomy' by the older birth data collection form, $586(21.9 \%)$ as 'first-degree tear/graze', 532 (19.9\%) as 'episiotomy', 389 (14.5\%) as 'second-degree tear', $52(1.9 \%)$ as 'other', $33(1.2 \%)$ as 'intact', and five with missing data on the birth record. The coding 'other' refers to an unspecified perineal tear, or vulval or perineal haematoma. Among the more recent data, 1588 out of 9916 women (16.0\%) were coded with severe perineal trauma by linked data but not by birth data. Birth data coding for these women reported 873 (55.0\%) as 'other', 395 (24.9\%) as 'second-degree tear', 276 (17.4\%) as 'first-degree tear', 43 (2.7\%) as 'intact', and one with missing birth data.

Among the more recent data, the distributions of maternal age, primiparity, instrumental birth, birthweight $\geq 4 \mathrm{~kg}$ or Asian maternal country of birth were similar for women identified with severe perineal trauma from birth data and women identified from linked data (Table 2). Discordant cases in which severe perineal trauma was identified by linked data but not by birth data also had similar distributions for primiparity (75.8\%), fewer women aged $26-35$ years $(62.7 \%)$, higher rates of instrumental delivery (45.0\%), fewer infants $\geq 4 \mathrm{~kg}$ (15.4\%) and fewer Asian-born women (20.4\%). However, using only birth data, $35.4 \%$ of women identified as having severe perineal trauma were coded with an episiotomy, compared with $40.2 \%$ in the linked data.
The adjusted risk estimates for maternal age, parity, instrumental delivery, birthweight and Asian country of birth were similar when severe perineal trauma was identified by birth data and by linked data (Table 2). In contrast, episiotomy was not significantly associated with severe perineal trauma when only birth data were analysed (aOR 1.02, 95\% Cl 0.97, 1.08), but was associated in linked data (aOR 1.33, 95\% Cl 1.27, 1.40). Analysis, depending on the type of delivery, revealed episiotomy as a risk factor for noninstrumental birth and protective for forceps delivery in both scenarios. However, among vacuum births, the aOR for episiotomy was 0.77 (95\% Cl 0.70, 0.85) when birth data were used, and nonsignificant (aOR 1.0195\% Cl 0.92, 1.11) using linked data.

\section{Discussion}

We have demonstrated that ascertaining severe perineal trauma rates from birth data alone results in lower reported rates compared with ascertainment from linked data. Because we had no validated dataset with which to compare our results, we cannot quantify if higher ascertainment may have been a result of false positive results within the linked data. However, we are reassured by a previous validation study, which reported that the combination of procedure or diagnosis codes from hospital data had a higher positive predictive value (PPV) of 99.7 than birth data alone (PPV $=75.7) .{ }^{13}$

Since the introduction of the revised birth data collection form in 2006, agreement between severe perineal trauma reporting by linked and birth data has improved. For birth data collected on the older version of the form, a woman with severe perineal trauma and episiotomy could not be counted in the severe perineal trauma group because documentation did not specify the tear type. In the older data, $40 \%$ of discordant cases were coded for 'both tear and episiotomy', and exclusion of this group would have partially contributed to lower reporting in the birth data compared with the linked data. However, even after the newer form was introduced, linked data still identified more cases of severe perineal trauma than birth

Table 1. Comparison of severe perineal trauma (SPT) coding by linked data, with different versions of birth data collection form, among vaginal births

\begin{tabular}{|c|c|c|c|c|c|c|}
\hline \multirow[b]{2}{*}{ Form } & & \multicolumn{2}{|c|}{ Linked data } & \multirow[b]{2}{*}{ Total (\%) } & \multirow[b]{2}{*}{ Agreement (\%) } & \multirow[b]{2}{*}{ Kappa (95\% CI) } \\
\hline & & $\begin{array}{c}\text { SPT recorded, } \\
n(\%)\end{array}$ & $\begin{array}{c}\text { SPT not recorded, } \\
n(\%)\end{array}$ & & & \\
\hline \multirow{3}{*}{$\begin{array}{l}\text { Earlier birth } \\
\text { data }\end{array}$} & SPT recorded & $5563(1.6)$ & $314(0.1)$ & $5877(1.7)$ & & \\
\hline & SPT not recorded & $2679(0.8)$ & 340843 (97.6) & $343522(98.3)$ & & \\
\hline & Total & $8242(2.4)$ & 341157 (97.6) & 349399 (100.0) & 99.1 & $0.78(0.78,0.79)$ \\
\hline \multirow{3}{*}{$\begin{array}{l}\text { More recent } \\
\text { birth data }\end{array}$} & SPT recorded & $8328(2.4)$ & $406(0.1)$ & $8734(2.5)$ & & \\
\hline & SPT not recorded & $1588(0.5)$ & 337481 (97.0) & 339069 (97.5) & & \\
\hline & Total & $9916(2.8)$ & 337887 (97.2) & $347803(100.0)$ & 99.4 & $0.89(0.89,0.89)$ \\
\hline
\end{tabular}


Table 2. Distributions of factors and association with severe perineal trauma (SPT) by data source, 2006-2011

\begin{tabular}{|c|c|c|c|c|c|}
\hline \multirow[b]{2}{*}{ Risk factor } & \multirow[b]{2}{*}{ Category } & \multicolumn{2}{|c|}{ Scenario 1: birth data only } & \multicolumn{2}{|c|}{ Scenario 2: linked data } \\
\hline & & $\begin{array}{l}\text { SPT recorded in } \\
\text { birth data, } \\
n=8734(2.5 \%)\end{array}$ & $\mathrm{aOR}(95 \% \mathrm{Cl})^{\mathrm{a}}$ & $\begin{array}{c}\text { SPT recorded } \\
\text { in hospital data, } \\
n=9916(2.8 \%)\end{array}$ & aOR $(95 \% \mathrm{Cl})^{\mathrm{a}}$ \\
\hline \multirow[t]{3}{*}{ Maternal age (years) $)^{b}$} & $\leq 25$ & $2102(24.1)$ & $0.85(0.81,0.90)$ & 2415 (24.4) & $0.88(0.84,0.92)$ \\
\hline & $26-35$ & 5759 (65.9) & Reference & $6479(65.3)$ & Reference \\
\hline & $>35$ & $873(10.0)$ & $0.79(0.73,0.85)$ & $1022(10.3)$ & $0.82(0.77,0.88)$ \\
\hline \multirow[t]{3}{*}{ Parity $^{b}$} & Primipara & $6582(75.4)$ & $3.56(3.37,3.76)$ & $7483(75.5)$ & $3.42(3.25,3.60)$ \\
\hline & Multipara & $2143(24.5)$ & Reference & 2422 (24.4) & Reference \\
\hline & Missing & $9(0.1)$ & na & $11(0.01)$ & na \\
\hline \multirow[t]{2}{*}{ Instrumental birth ${ }^{b}$} & Yes & 3658 (41.9) & $2.30(2.18,2.43)$ & $4182(42.2)$ & $2.10(1.99,2.20)$ \\
\hline & No & $5076(58.1)$ & Reference & $5734(57.8)$ & Reference \\
\hline \multirow[t]{3}{*}{ Birthweight ${ }^{b}$} & $\geq 4 \mathrm{~kg}$ & $1589(18.2)$ & $2.45(2.31,2.60)$ & $1753(17.7)$ & $2.33(2.21,2.47)$ \\
\hline & $<4 \mathrm{~kg}$ & $7144(81.8)$ & Reference & 8161 (82.3) & Reference \\
\hline & Missing & $1(<0.1)$ & na & $2(<0.1)$ & na \\
\hline \multirow[t]{3}{*}{ Country of birth ${ }^{b}$} & Asian & $2496(28.6)$ & $2.28(2.17,2.40)$ & $2761(27.8)$ & $2.13(2.03,2.23)$ \\
\hline & Non-Asian & 6175 (70.7) & Reference & 7077 (71.4) & Reference \\
\hline & Missing & $63(0.7)$ & na & $78(0.8)$ & na \\
\hline \multirow[t]{3}{*}{ Episiotomy ${ }^{\mathrm{C}}$} & Yes & 3091 (35.4) & $1.02(0.97,1.08)$ & 3986 (40.2) & $1.33(1.27,1.40)$ \\
\hline & No & $5642(64.6)$ & Reference & 5930 (59.8) & Reference \\
\hline & Missing & $1(<0.01)$ & na & 0 & na \\
\hline
\end{tabular}

95\% Cl = 95\% confidence interval; aOR = adjusted odds ratio; na = not applicable

a Adjusted for all factors in table

b Identified by birth data

c Identified by birth data alone for scenario 1; identified if recorded in birth or in hospital data for scenario 2

data. We cannot determine the reason, but this finding may be influenced by coding practices and when the data were recorded.

After the revised form was introduced, severe perineal trauma reporting spiked for 2007 birth data; however, this spike was not maintained and the difference between linked and birth data remained fairly constant over the period to 2011. The different recorded rates by data source affected the change in incidence over time, with birth data reflecting a much larger increase than linked data. We would recommend that if a researcher did not have access to linked data and was using birth data, rates of severe perineal trauma should be determined from data collected on the recent form, with acknowledgement that underascertainment is still likely.

Any differences in distributions of discordant cases were not influential enough to have a major effect on the adjusted risk estimates for parity, instrumental delivery, infants $\geq 4 \mathrm{~kg}$ and Asian country of birth. However, episiotomy was not significantly associated with severe perineal trauma using birth data alone, but was associated in linked data. This suggests that, by using birth data alone, researchers may underestimate the overall association of episiotomy with severe perineal trauma.
Although two datasets were compared, we did not have access to clinical details in the original medical records, which could have provided us with the most accurate information (or the 'gold standard'). This is a limitation of this study. We could not quantify the extent to which higher ascertainment within the linked data represented a real increase or increased reporting. Strengths of this study include the use of population data, thus avoiding sampling bias and allowing investigation of a relatively rare outcome.

\section{Conclusion}

We have shown that the use of linked data results in higher ascertainment of severe perineal trauma than birth data, reflected in higher overall rates. With the revision of the birth data collection form, allowing separate recording of perineal tears and episiotomy, agreement between rates calculated from birth data and linked data improved; however, possible underreporting of severe perineal trauma by birth data remained. These differences have an impact when describing the changes in rates of severe perineal trauma over time. The differences in distributions of episiotomy as reported by birth data compared with linked data translated into significant differences in adjusted risk estimates. 


\section{Acknowledgements}

The authors wish to acknowledge the NSW Ministry of Health for access to the population health data, and the NSW Centre for Health Record Linkage for linking the datasets. AA is supported by the Dr Albert S McKern Research Scholarship and JF by an Australian Research Council Future Fellowship.

\section{Competing interests}

None declared

\section{Author contributions}

AA participated in the study design, performed the data analysis and interpretation, and was the main author of the manuscript. JF participated in the study design and in the interpretation of the data, and reviewed and edited the manuscript.

\section{References}

1. Priddis H, Dahlen H, Schmied V. Women's experiences following severe perineal trauma: a meta-ethnographic synthesis. J Adv Nurs. 2013;69(4):748-59.

2. Fornell EU, Matthiesen L, Sjödahl R, Berg G. Obstetric anal sphincter injury ten years after: subjective and objective long term effects. BJOG. 2005;112(3):312-6.

3. Wegnelius G, Hammarström M. Complete rupture of anal sphincter in primiparas: long-term effects and subsequent delivery. Acta Obstet Gynecol Scand. 2011;90(3):258-63.

4. Ampt AJ, Ford JB, Roberts CL, Morris JM. Trends in obstetric anal sphincter injuries and associated risk factors for vaginal singleton term births in New South Wales 2001-2009. Aust N Z J Obstet Gynaecol. 2013;53(1):9-16

5. Jangö H, Langhoff-Roos J, Rosthøj S, Sakse A. Modifiable risk factors of obstetric anal sphincter injury in primiparous women: a population-based cohort study. Am J Obstet Gynecol. 2014;210(1):59e1-e6.

6. Ismail S, Puyk B. The rise of obstetric anal sphincter injuries (OASIS): 11-year trend analysis using patient episode database for Wales (PEDW) data. J Obstet Gynaecol. 2014;34(6):495-8.

7. Gurol-Urganci I, Cromwell D, Edozien L, Mahmood TA, Adams EJ, Richmon DH, et al. Third- and fourth-degree perineal tears among primiparous women in England between 2000 and 2012: time trends and risk factors. BJOG. 2013;120(12):1516-25.

8. Räisänen S, Vehviläinen-Julkunen K, Gissler M Heinonen S. The increased incidence of obstetric anal sphincter rupture - an emerging trend in Finland. Prev Med. 2009;49(6):535-40.
9. Australian Institute of Health and Welfare. Towards national indicators of safety and quality in health care. Canberra: Australian Institute of Health and Welfare; 2009 [cited 2015 Jun 10]. Available from: www.aihw.gov.au/ publication-detail/?id=6442468285

10. Räisänen S, Selander T, Cartwright R, Gissler M, Kramer MR, Laine K, Heinonon S. The association of episiotomy with obstetric anal sphincter injury - a population based matched cohort study. PLoS One. 2014;9(9):e107053.

11. Bell JC, Ford JB, Cameron CA, Roberts CL. The accuracy of population health data for monitoring trends and outcomes among women with diabetes in pregnancy. Diabetes Res Clin Pract. 2008;81(1):105-9.

12. Lain SJ, Hadfield RM, Raynes-Greenow CH, Ford JB, Mealing NM, Algert CM, Roberts CL. Quality of data in perinatal population health databases: a systematic review. Med Care. 2012;50(4):e7-e20.

13. Roberts CL, Bell JC, Ford JB, Morris JM. Monitoring the quality of maternity care: how well are labour and delivery events reported in population health data? Paediatr Perinat Epidemiol. 2008;23(2):144-52.

14. Ampt AJ, Ford JB, Taylor LK, Roberts CL. Are pregnancy outcomes associated with risk factor reporting in routinely collected perinatal data? NSW Public Health Bulletin. 2013;24(2):65-9.

15. Baghestan E, Børdahl PE, Rasmussen SA, Sande AK, Lyslo I, Solvang I. A validation of the diagnosis of obstetric sphincter tears in two Norwegian databases, the Medical Birth Registry and the Patient Administration System. Acta Obstet Gynecol Scand. 2007;86(2):205-9.

16. Kuklina EV, Whiteman MK, Hillis SD, Jamieson DJ, Meikle SF, Posner SF, Marchbanks PA. An enhanced method for identifying obstetric deliveries: implications for estimating maternal morbidity. Mat Child Health J. 2008;12(4):469-77.

17. McLoughlin V, Millar J, Mattke S, Franca M, Jonsson PM, et al. Selecting indicators for patient safety at the health systems level in OECD countries. Int J Qual Health Care. 2006 Sep;18 Suppl 1:14-20.

18. Chen JS, Ford JB, Ampt A, Simpson JM, Roberts CL. Characteristics in the first vaginal birth and their association with mode of delivery in the subsequent birth. Paediatr Perinat Epidemiol. 2013;27(2):109-17.

19. Ford JB, Bentley JP, Morris JM, Roberts CL. Factors associated with changes into public or private maternity care for a second pregnancy. Aust Health Rev. 2013;37(4):495-500.

20. Dahlen H, Priddis H, Schmied V, Sneddon A, Kettle C, Brown C, Thornton C. Trends and risk factors for severe perineal trauma during childbirth in New South Wales between 2000 and 2008: a population-based data study. BMJ Open. 2013;3(5):3:e002824. 
21. Robson SJ, Laws P, Sullivan EA. Adverse outcomes of labour in public and private hospitals in Australia: a population-based descriptive study. Med J Aust. 2009;190(9):474-7.

22. Demand and Performance Evaluation Branch. Centre for Epidemiology and Health. Perinatal data collection manual - 2011 edition. Sydney: NSW Department of Health 2010.

23. National Centre for Classification in Health. The international statistical classification of diseases and related health problems, tenth revision, Australian modification 7th edition. Tabular list of diseases. Sydney: The University of Sydney; 2010.
24. National Centre for Classification in Health. The international statistical classification of diseases and related health problems, tenth revision, Australian modification 7th edition. Tabular list of interventions. Sydney: The University of Sydney; 2010.

25. Pergialiotis V, Vlachos D, Protopapas A, Pappa K, Vlachos $\mathrm{G}$. Risk factors for severe perineal lacerations during childbirth. Int J Gynecol Obstet. 2014;125(1):6-14.

\section{Copyright: (c)(ㅇ) (2)}

(C) 2015 Ampt and Ford. This article is licensed under the Creative Commons Attribution-NonCommercial-ShareAlike 4.0 International Licence, which allows others to redistribute, adapt and share this work non-commercially provided they attribute the work and any adapted version of it is distributed under the same Creative Commons licence terms. See: www.creativecommons.org/licenses/by-nc-sa/4.0/ 